\title{
Postural Orthostatic Tachycardia Syndrome following Open Thoracoabdominal Aortic Aneurysm repair
}

\author{
Lucy Manuel ${ }^{1}$, Laura Fong ${ }^{1}$, Andrew Mamo ${ }^{1}$, Ramon Varcoe ${ }^{1}$, Wilfred Saw ${ }^{1}$, and Peter \\ Grant $^{1}$ \\ ${ }^{1}$ Prince of Wales Hospital and Community Health Services
}

October 1, 2020

\begin{abstract}
Postural Orthostatic Tachycardia Syndrome (POTS) is a variant of cardiovascular autonomic disorder characterised by an excessive heart rate on standing and orthostatic intolerance. We present a rare case of a 38-year old man who underwent open repair of a thoracoabdominal aortic aneurysm (TAAA) for a chronic Stanford type B aortic dissection whose recovery was complicated by POTS. He was received blood transfusions, and was commenced on metoprolol, fludrocortisone and ivabradine with significant improvement in his symptoms.
\end{abstract}

\section{Introduction}

Postural Orthostatic Tachycardia Syndrome (POTS) is a common, though not well understood, variant of cardiovascular autonomic disorder characterised by an excessive heart rate on standing and orthostatic intolerance. It typically affects younger individuals, aged 15-45 years, with an $80 \%$ female and $>90 \%$ Caucasian predominance $[1,2]$.

\section{Case report}

We present the case of a 38-year old Brazilian man who presented with a 4-day history of chest pain radiating to his abdomen and back. He was subsequently diagnosed with an extensive Stanford type B aortic dissection originating at the base of the left subclavian artery with extension into the iliac arteries. With the exception of the left renal artery, all visceral branches were supplied by the true lumen. He was managed conservatively with strict blood pressure control and discharged home. On serial CT aortogram 8 weeks later, the dissection appeared stable, however, the diameter of the distal thoracic descending aorta had increased to $64 \mathrm{~mm}$ from $59 \mathrm{~mm}$. His aortic arch was deemed to be unsuitable for stent grafting due to the acute angle from the arch (60 degree angulation) into the aneurysm, resulting in a Gothic arch; and he was planned for open surgical repair.

A further 3 months later, prior to planned repair, the patient presented with severe left sided chest pain. A repeat CT aortogram demonstrated a fusiform dilatation of the distal arch and descending thoracic aorta measuring $73 \mathrm{x} 69 \mathrm{~mm}$ wide (Figure 1) with interval dilation of the false lumen. The decision was made to proceed with an open thoracoabdominal aortic aneurysm (TAAA) repair utilising cardiopulmonary bypass with deep hypothermic circulatory arrest. A pre-operative cerebrospinal drain was placed to decrease the risk of spinal ischaemia throughout the operation. The patient was positioned in the left lateral position with a thoracoabdominal incision made through the fifth intercostal space. Retroperitoneal dissection revealed vessels of good calibre and an extensive TAAA particularly in the upper thorax (Figure 2). Peripheral cardiopulmonary bypass was instituted via the left femoral artery and vein utilising an $8 \mathrm{~mm}$ Dacron graft and $\mathrm{Y}$ connector, and a $25 \mathrm{Fr}$ venous cannula respectively. The patient was cooled to 18 degrees Celsius and placed in Trendelenburg positioning for clamping of the mid thoracic aorta. 
The aneurysm was incised and the dissection flap unroofed with a $2 \mathrm{~cm}$ cuff created for the proximal anastomosis using a $26 \mathrm{~mm}$ Dacron graft. Arterial inflow was established via an $8 \mathrm{~mm}$ sidearm, with clamping of the femoral line and subsequent deairing of the cerebral circulation and rewarming of the patient. The Dacron graft was then clamped proximal to the arterial inflow and femoral bypass reinstated with the clamp moved to just above the diaphragm. Multiple small intercostal arteries were oversewn, with two large intercostals at T10 controlled with Fogarty catheters and anastomosed utilising separate 8mm Dacron grafts. The cross clamp was moved to just above the renal arteries, allowing a Carrel patch containing the coeliac trunk and superior mesenteric arteries to be fashioned. Continuous perfusion to the superior mesenteric artery was provided via a $12 \mathrm{Fr}$ cannula. The femoral inflow was clamped, and the abdominal aortic clamp removed, with fenestration of the aorta to ensure the left femoral artery was supplied by the true lumen. A size $28 \mathrm{~mm}$ Dacron graft was anastomosed to the suprarenal aorta, a clamp placed superior to the suture line, and femoral inflow restored. The Carrel patch containing coeliac trunk and superior mesenteric vessels was anastomosed to the anterior aspect of the graft.

The graft was positioned through the aortic opening in the diaphragm and the 8mm Dacron graft supplying the intercostals anastomosed towards the posterolateral side of the graft. The $26 \mathrm{~mm}$ and $28 \mathrm{~mm}$ Dacron grafts were anastomosed in the mid thoracic aorta with deairing and clamping of femoral inflow. The patient was weaned from bypass without difficulty and the venous line removed with repair of the femoral vein. The femoral and thoracic $8 \mathrm{~mm}$ Dacron inflow grafts were ligated and divided, with repair of the diaphragm and costal margins undertaken. The bypass time was 312 minutes and upper body circulatory arrest time 39 minutes. He was transferred to intensive care in a stable condition with systolic blood pressure targets of 90-100 $\mathrm{mmHg}$. His immediate post-operative course was complicated by vasoplegia, requiring noradrenaline, and coagulopathy requiring massive transfusion.

The patient was initially agitated and unable to obey commands. He became febrile on day 6 post-operatively and was diagnosed with a ventilator acquired pneumonia and commenced on tazocin and vancomycin. He was extubated on day 8 with improvement in neurology, however, required reintubation due to respiratory distress later that day. He remained febrile, with surgical wound dehiscence and escalation of his antibiotics to meropenem and vancomycin. Despite adequate treatment of his pneumonia, with successful extubation on day 10, and wound dehiscence with antibiotics and surgical debridement, the patient had persistent tachycardia up to $180 \mathrm{bpm}$ without haemodynamic compromise. Post-operative transthoracic echocardiography revealed moderate global impairment with trivial pericardial effusion. Postural telemetry (Figure 3), demonstrated a sitting heart rate of $101 \mathrm{bpm}$ which increased to $144 \mathrm{bpm}$ on standing and $164 \mathrm{bpm}$ on mobilisation without incrementation of the blood pressure (110/54mmHg sitting to $100 / 67 \mathrm{mmHg}$ standing), consistent with POTS. The patient was commenced on metoprolol, fludrocortisone and ivabradine with improvement in his tachycardia. He received multiple blood transfusions to increase his haemoglobin from 90 to $110 \mathrm{~g} / \mathrm{L}$. He was discharged home 6 weeks after his operation with no disability.

\section{Comment}

This case highlights an unusual cause for persistent post-operative sinus tachycardia in a young patient, with only $2.8 \%$ of POTS cases being attributed to surgery [2]. The diagnosis of POTS is based on three factors. Firstly, a clinical syndrome characterised by symptoms of light-headedness, blurry vision, palpitations, exercise intolerance and fatigue. Secondly, an increase of more than 30bpm ([?] 40bpm in 12-19 years) when the person stands up from recumbent position. Thirdly, absence of orthostatic hypotension $[3,4,5]$. The onset of POTS may be precipitated by typical immunological stressors such as surgery, viral infection, vaccination, trauma, pregnancy or psychological stress [1].

Non-pharmacological treatment remains first-line and involves increasing aerobic exercise, lower extremity strengthening, increasing salt and fluid intake, psychological training for anxiety or pain management and education. Pharmacological therapy is considered on an individual basis and consists of beta-blockers to blunt orthostatic increases in heart rate, alpha-adrenergic agents to increase peripheral vascular resistance, mineralocorticoids to increase blood volume and serotonin reuptake inhibitors to improve serotonin regulation $[4]$. 
There has only been one other case report in the literature reporting on the development of POTS following thoracic surgery, involving a repair of an aortic coarctation [6]. Similarly, they identified potential risk factors including surgery, prolonged bed rest, and use of antihypertensives that are known to worsen orthostatic intolerance (amlodipine and valsartan). Additional risk factors in our case include reduced oral intake, anaemia, autonomic insufficiency and poor sleep [1]. Correct assessment of post-operative tachycardia including postural telemetry is the key to identifying this condition and its successful management.

\section{Acknowledgments}

Nil

\section{Disclosures}

Nil

\section{Ethics Statement}

The case was discussed with the institutional review board and deemed exempt from review. Consent for the patient was obtained for the purpose of publishing the case

\section{References}

1. Fedorowski A. Postural orthostatic tachycardia syndrome: clinical presentation, aetiology and management. J Intern Med. 2019;285(4):352-66.

2. Boris JR, Bernadzikowski T. Demographics of a large paediatric Postural Orthostatic Tachycardia Syndrome Program. Cardiol Young. 2018;28(5):668-74.

3. Tahir F, Bin Arif T, Majid Z, Ahmed J, Khalid M. Ivabradine in Postural Orthostatic Tachycardia Syndrome: A Review of the Literature. Cureus. 2020;12(4):e7868-e.

4. Johnson JN, Mack KJ, Kuntz NL, Brands CK, Porter CJ, Fischer PR. Postural orthostatic tachycardia syndrome: a clinical review. Pediatr Neurol. 2010;42(2):77-85.

5. Zadourian A, Doherty TA, Swiatkiewicz I, Taub PR. Postural Orthostatic Tachycardia Syndrome: Prevalence, Pathophysiology, and Management. Drugs. 2018;78(10):983-94.

6. Fernex L, Coeytaux A, Rochat T, Karaca S, Perrig S, Burri H, et al. Postural orthostatic tachycardia syndrome after surgical correction of an aortic coarctation: a case report. J Med Case Rep. 2012;6:241.

\section{Figures}

Figure 1: April $2020 \mathrm{CT}$ aortogram demonstrating a large $72.7 \mathrm{~mm}$ x $68.6 \mathrm{~mm}$ fusiform aneurysm- A: axial slices, B: coronal slices, C: sagittal slices, D: 3D reconstruction

Figure 2: Intraoperative photographs demonstrating a 70mm thoracoabdominal aortic aneurysm

Figure 3: A: Telemetry demonstrating a heart rate of $164 \mathrm{bpm}$ on mobilization, B: Telemetry demonstrating a heart rate of $101 \mathrm{bpm}$ on returning to sitting position 
Figure 1

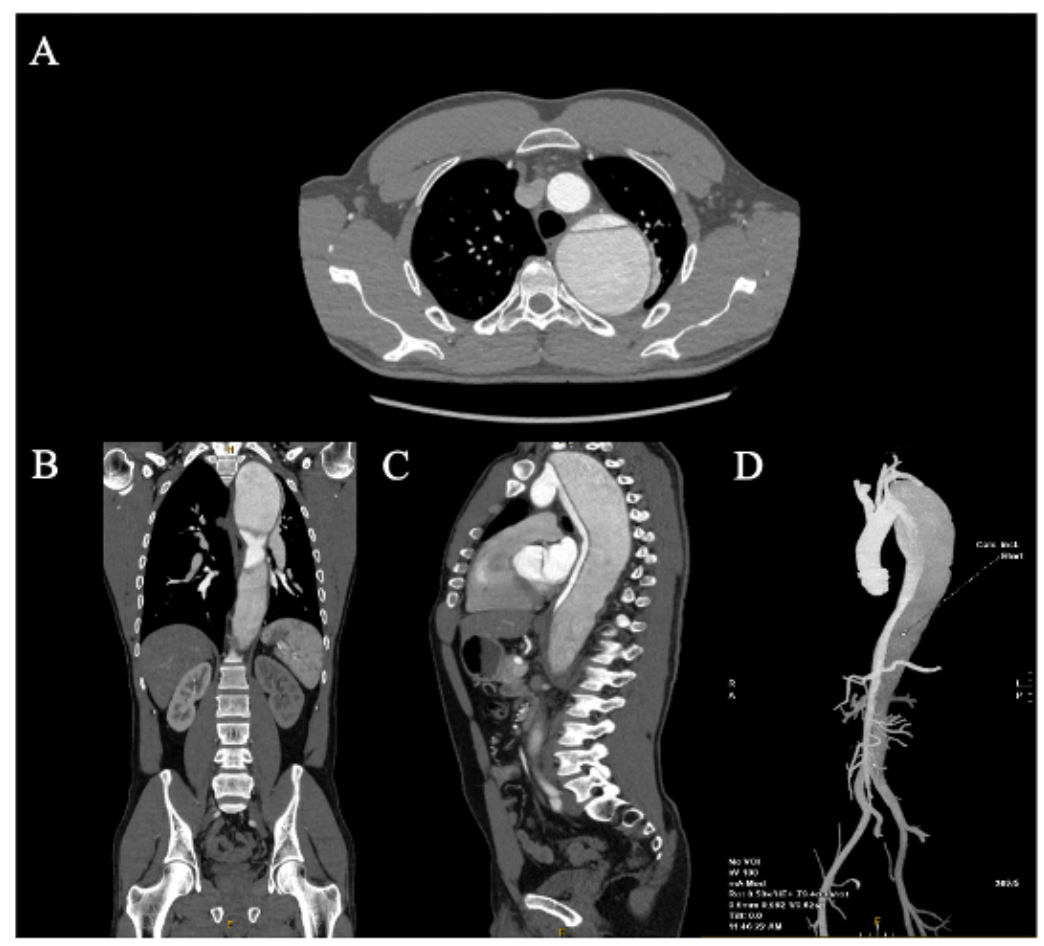

Figure 2
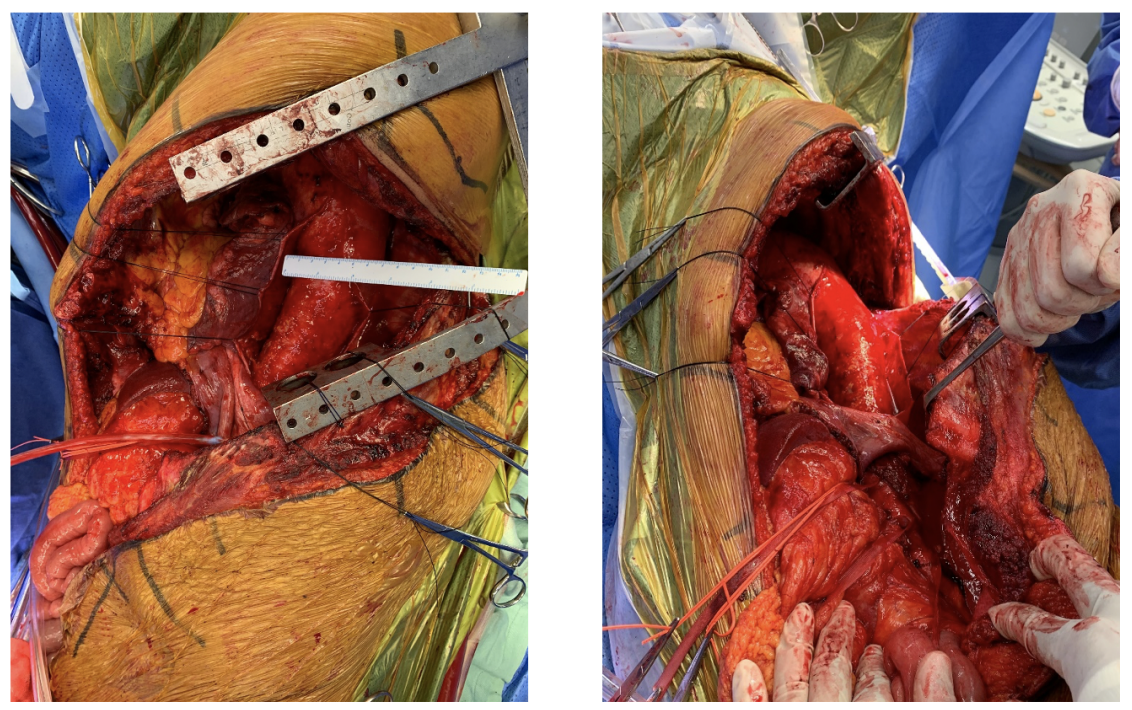
Figure 3

A Vitals:

ובבטנטן

Vitals:
HR 164

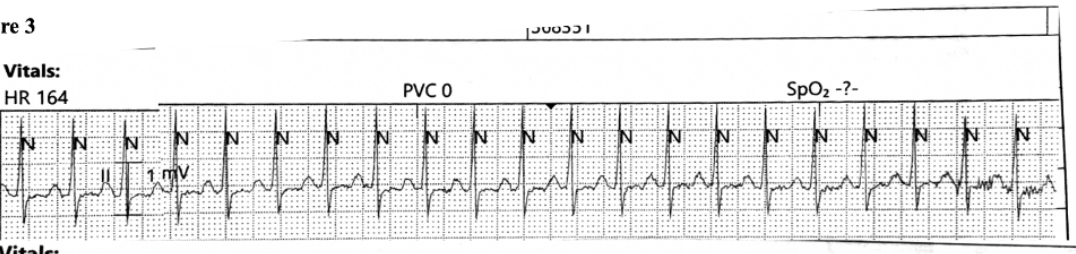

B Vitals:

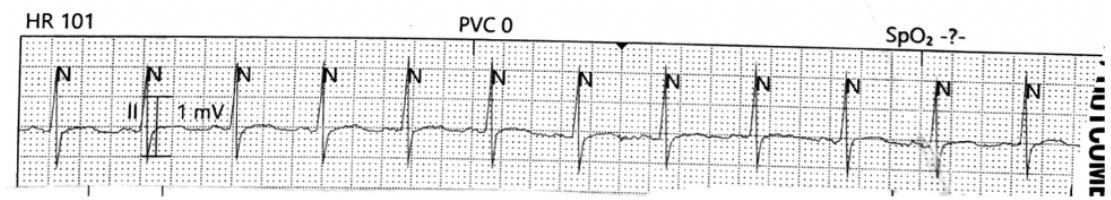

